\title{
Solutions of Minimal Period for a Semilinear Wave Equation $\left({ }^{*}\right)$.
}

Ann. Mat. Pura e Appl., (IV), CLV (1989), pp. 271-284

AdDolorata SaLVATore

Let $\bar{u}(t, x)$ be a solution of the following wave equation

$$
u_{t t}-u_{x x}+g(u)=0 \quad t \in \mathbb{R}, x \in[0, \pi]
$$

under boundary and periodicity conditions

$$
\left\{\begin{array}{l}
u(t, 0)=u(t, \pi)=0 \\
u(t, x)=u(t+T, x)
\end{array} \quad t \in \mathbb{R}, x \in[0, \pi] .\right.
$$

In the proof of Theorem 1.7 we say that if $\bar{u}(t, x)$ is $T / n$-periodic in $t$, there exist at least $n-1$ points conjugate to 0 in $] 0, T[$, namely $\{T / n, 2 T / n, \ldots,(n-1) T / n\}$. However this is true if $\bar{u}(t, x)$ verifies the additional condition $\bar{u}(t, 0)=\bar{u}(t, \pi / n)=$ $=\ldots=\bar{u}(t,(n-1) \pi / n)=\bar{u}(t, \pi)=0$.

Therefore the proof of the relation between $m(\bar{v})$ and $O(\bar{u})$ stated in Theorem 1.7 holds if we modify the definition of $O(\bar{u})$ as follows:

«O( $O(\bar{u})$ is the greatest integer $n$ such that $\bar{u}(t, x)$ is $T / n$-periodic in $t$ and

$$
\bar{u}(t, 0)=\bar{u}(t, \pi / n)=\ldots=\bar{u}(t,(n-1) \pi / n)=\bar{u}(t, \pi)=0 . \text { » }
$$

That implies that the solution $\bar{u}$ found in Theorem 0.3 and 0.4 in general does not have minimal period $T$, but it verifies a "minimality " condition in the variables $(t, x)$, more precisely it does not exist any $n \in \mathbb{N}$ such that $\bar{u}(t, x)$ is $T / n$-periodic in $t$ and $\bar{u}(t, 0)=\bar{u}(t, \pi / n)=\ldots=\bar{u}(t,(n-1) \pi / n)=\bar{u}(t, \pi)=0$.

(*) Entrata in Redazione il 6 giugno 1990. 